\title{
Revue
}

\section{Utilisation du blé et des céréales dans la ration des vaches laitières}

\author{
F. LAURENT \\ Université Nancy $I$, \\ Sciences et Techniques des Productions Animales E.N.S.A.I.A.-I.N.P.L. \\ 2, avenue de la Forêt de Haye, B.P. 172, 54505 Vandouvre-lès-Nancy
}

\begin{abstract}
Résumé
Le blé est actuellement peu utilisé dans les rations des vaches laitières. Sa teneur en amidon est proche de celle du maïs mais ses teneurs en protéines et en azote soluble sont plus élevées. La dégradation du blé et de l'orge dans le rumen est plus rapide que celle du maïs, la différence étant plus forte pour les protéines que pour l'amidon. Le blé est en général plus favorable que le maïs ou le sorgho à la production d'acide propionique dans le rumen; le roulage ou le broyage des grains accentuent cette tendance.

Comparé au maïs le blé ne diminue pas l'ingestion tant que la teneur en concentrés est inférieure à 50 p. 100 dans la ration ou si le concentré est mélangé au fourrage. Les essais de comparaison entre céréales, souvent réalisés avec du foin de luzerne et sur des périodes courtes, montrent que la nature de la céréale intervient peu sur la production et la composition du lait tant que l'on est sous le seuil de 50 p. 100 de concentrés dans la ration. Le blé entier est en général moins bien valorisé que le blé aplati ou le blé broyé. Une adaptation progressive à des rations contenant des quantités importantes de blé permet d'éviter les accidents sanitaires.
\end{abstract}

Mots clés: Blé, céréales, vaches laitières, lait.

\section{Introduction}

Des considérations techniques et économiques ont jusqu'à présent limité l'utilisation du blé comme aliment concentré dans la ration des vaches laitières. A mesure qu'augmente le potentiel de production, les stocks gonflent sur le marché mondial entraînant à la baisse les prix et incitant à la recherche de nouveaux débouchés. Le développement de l'utilisation de blé dans la ration des vaches laitières pourrait constituer un de ces marchés.

Des revues bibliographiques récentes permettent de comparer diverses céréales entre elles (Thivend, 1981 ; Orskov, 1986 ; OWens et al., 1986 ; RoOney \& PflugfelDER, 1986 ; THEURER, 1986) mais ces travaux privilégient le plus souvent des comparai-

Cette étude a été réalisée dans le cadre d'une convention I.T.E.B.-Université Nancy I. 
sons maïs-sorgho et n'intègrent pas ou très rarement les aspects liés à l'utilisation du blé.

L'objectif de cette revue est donc de rappeler rapidement les caractéristiques essentielles du blé comparativement aux autres céréales, de préciser les différences de digestibilité mesurées entre le blé et les autres céréales, enfin de définir à partir des rares essais réalisés sur vaches laitières les conditions d'utilisation de cette céréale.

\section{A. Rappel sur la composition des graines de céréales}

Le blé est caractérisé par sa faible teneur en cellulose brute $(2,3$ p. 100 soit l'équivalent du maïs mais moitié moins que l'orge), sa faible teneur en lipides (environ 2 p. 100 comme l'orge contre 4 p. 100 au maïs) (données UCAAB, 1984).

Les teneurs en amidon sont fortes (59 p. 100) voisines de celles du maïs (63 p. 100) et du sorgho $(60$ p. 100$)$. Les proportions d'amylose $(28 \mathrm{p} .100)$ et d'amylopectine (72 p. 100) dans l'amidon total sont les mêmes pour le blé et le maîs, les grains d'amidon ont le même diamètre moyen $(15 \mu)$ mais la dispersion est plus forte pour le blé que pour le maïs (Swinkels, 1985). L'amidon de blé est plus sensible à la chaleur (température de gélatinisation plus basse), plus soluble dans l'eau (viscosité nettement inférieure) et contient plus de protéines $(0,4 \mathrm{p}$. 100) que l'amidon de maïs $(0,3 \mathrm{p} .100)$. Les teneurs en sucres solubles, en sucres réducteurs et en pentosanes sont peu différentes entre le blé et l'orge ; le maïs et le sorgho sont moins riches en glucides facilement dégradables dans le rumen (Thivend, 1981 ; Henry, 1985).

La répartition des protéines dans le grain de céréales n'est pas uniforme : la couche à aleurones comporte 30 à $35 \mathrm{p}$. 100 de protéines, le germe 35 à $40 \mathrm{p}$. 100 mais le péricarpe et l'albumen amylacé respectivement 6 à 7 p. 100 et 6 à 9 p. 100 (PoPINEAU, 1985). Ces protéines sont de nature diverse : protéines de structures, enzymes, protéines de réserves.

Le blé renferme plus de protéines solubles (15 p. 100 albumines + globulines, essentiellement des enzymes), que le maïs (6 p. 100) autant que le sorgho (16 p. 100) et moins que l'orge (25 p. 100). L'avoine est exceptionnellement riche en protéines solubles (67 p. 100). Hormis le cas particulier de l'avoine (9 p. 100), les teneurs en prolamines qui sont des protéines de réserves sont comprises entre $40 \mathrm{p} .100$ (blé) et 55 p. 100 (maïs). Chez le blé, les prolamines ou gliadines présentent la propriété unique de pouvoir former après hydratation une masse cohérente insoluble et viscoélastique, propriété mise à profit dans la panification.

Chez le sorgho, les prolamines ou kafirines forment des couches entrecroisées qui protègent l'amidon de la zone farineuse et expliquent sa faible digestibilité relative (RoONey \& Pflugfelder, 1986). Les gluténines qui constituent des protéines de structure sont surtout localisées dans la couche à aleurones : elles constituent 46 p. 100 des protéines totales dans le grain de blé, 39 p. 100,38 p. 100 , et 23 p. 100 respectivement pour le mais, le sorgho et l'orge (Cheftel et al., 1985).

Sur le plan qualitatif, les protéines du mais et du sorgho sont pauvres en lysine, en méthionine + lysine, en arginine et en tryptophane. L'avoine présente les teneurs les plus élevées pour tous les acides aminés cités sauf la méthionine. Les premiers acides aminés limitants chez le blé sont la lysine, la thréonine et la valine mais le blé est plus riche en lysine que le maïs. 


\section{B. Digestion des graines de céréales}

\section{Au niveau du rumen}

\section{a) Dégradation de l'amidon}

La composition et la structure physique des graines, les interactions amidonprotéines, la forme de présentation des aliments, la présence de certains composés sont autant de variables affectant la digestibilité de l'amidon. La digestibilité est inversement proportionnelle à la teneur en amylopectine (Rooney \& Pflugfelder, 1986) mais elle est réduite par la présence de tannins, de lectines et de phytates (Rooney \& PflugfelDER, 1986) : les échelles de dégradabilité pour les amidons isolés à partir des divers grains (mesures de sensibilité à l'amylase bactérienne) montrent qu'il y a peu de différences entre amidon de maïs, de blé, d'orge et de sorgho, les proportions d'amylose et d'amylopectine étant identiques dans l'amidon de ces différentes céréales.

In sacco, les amidons de blé, d'orge, d'avoine sont dégradés beaucoup plus rapidement (40 à $60 \mathrm{p} .100 / \mathrm{h})$ que ceux du maîs et du sorgho ( 15 à $30 \mathrm{p} .100 / \mathrm{h})$ (Sauvant \& Michalet-Doreau, 1988). Les différences ont tendance à s'atténuer avec le temps : ainsi DoReAu (1988) rapporte qu'après $4 \mathrm{~h}$ d'incubation in sacco, 50 p. 100 de l'amidon de maïs a disparu contre $85 \mathrm{p} .100$ pour l'orge ou le blé. Après $24 \mathrm{~h}$, la totalité de l'amidon du blé ou de l'orge a été dégradée mais il reste 5 à $10 \mathrm{p} .100$ de l'amidon de maïs. Pour Thivend (1981) ces variations de vitesse de dégradation de l'amidon expliquent une part importante des différences enregistrées entre les cinétiques de dégradation de la matière sèche de l'orge et du blé d'une part et celle du mais d'autre part.

Dans les rares essais de comparaison directe entre céréales conduits in vivo (revue de Theurer, 1986) la digestibilité, dans le rumen, de l'amidon d'orge (93 p. 100) est supérieure à celle de l'amidon de maïs (73 p. 100) ou de sorgho (66 p. 100). In vivo, en moyenne, 90 à 95 p. 100 de l'amidon des grains d'orge, d'avoine ou de blé sont dégradés dans le rumen (Waldo, 1973 ; Orskov, 1986). Pour l'amidon des grains de maïs ou de sorgho le taux de dégradabilité moyen est compris entre 75 et 80 p. 100 (WALDO, 1973 ; OWENS et al., 1986).

Dans le rumen, pour l'orge, la revue de WaLDo (1973) montrait qu'il y a peu de variations de la dégradabilité (de l'ordre de $2,5 \mathrm{p} .100$ ) en fonction de la variété, de l'espèce animale (bovine ou ovine), du pourcentage dans la ration : la très forte digestion de l'amidon du grain d'orge explique sans doute l'absence de différence. Pour le maïs, la variabilité est au contraire très importante et atteint 16 p. 100 (revue de WALDO, 1973) ; la digestibilité dépend de l'origine variétale : elle est plus forte chez les ovins que chez les bovins (WALDo, 1973), et pourrait être liée au différentiel de vitesse de transit entre les animaux (Orskov, 1986). Il ne paraît pas y avoir de limite physiologique à la dégradation des amidons dans le rumen (Orskov, 1986).

\section{b) Dégradation des protéines}

En fait, les différences de digestibilité entre céréales ne sont pas liées à la taille ou à la structure du grain d'amidon ou même à sa composition. Le facteur déterminant serait à rechercher dans la nature et la composition des protéines (RoONEY \& PflugfelDER, 1986). En particulier, l'endosperme périphérique (couche à aleurones) est une 
zone riche en protéines qui est plus résistante et qui assure une «protection » aux zones amylacées sous-jacentes : la matrice protéique (glutélines) englobant les grains d'amidon peut être continue à ce niveau alors qu'elle présente une structure discontinue, donc plus fragile dans la zone farineuse (Gallant \& Bouchet, 1986).

Des écarts importants sont donc obtenus lors de la comparaison de la dégradabilité des protéines qui permet de discriminer deux groupes. En sachets de nylon, après $15 \mathrm{~h}$ de séjour dans le rumen 65,5 p. 100 des protéines de blé sont dégradées alors que pour le maïs la dégradabilité des protéines n'atteint que 28,8 p. 100 (NALSEN et al., 1987 c). En R.F.A. les valeurs moyennes de dégradabilité théorique des protéines dans le rumen sont de 65 p. 100 pour le maïs et 85 p. 100 pour le blé, l'orge ou l'avoine (RoHr, 1986). Dans le système P.D.I., VÉRITÉ et al. (1987) proposent des valeurs moyennes de dégradabilité théorique (blé 0,73 , orge 0,74 , avoine 0,77 , maïs 0,44 , sorgho 0,45 ) mais insistent sur la varibilité des résultats publiés.

Les mesures in vivo de SPICER et al. (1983) montrent que les protéines du maïs et du sorgho présentent une dégradabilité minimale plus faible (49 et $43 \mathrm{p}$. 100) que celle enregistrée pour l'orge (63 p. 100). Pour PonCET (cité par DoREau, 1988) la dégradabilité dans le rumen de l'azote du maïs est beaucoup plus faible que celle de l'orge (38 p. 100 contre 95 p. 100 ).

\section{c) Produits terminaux}

Les amidons des différentes céréales n'ont pas le même impact sur le mélange des A.G.V. dans le rumen. Des études comparatives ont été menées par Orskov (1986) avec des agneaux recevant les diverses céréales comme seule ration. Les résultats montrent que le rapport $\mathrm{C} 2 / \mathrm{C} 3$ le plus faible $(1,22)$ est obtenu avec du maïs grain entier alors que les valeurs enregistrées pour l'orge, le blé et l'avoine sont respectivement de $1,74,1,62$ et 3,49 . Sous forme broyée dans ces mêmes conditions le blé est plus favorable à la production de $\mathrm{C} 3$ (rapport $\mathrm{C} 2 / \mathrm{C} 3$ de 0,8 ) que le maïs $(0,99)$ et l'avoine $(1,42)$. Chez la vache laitière, pour des apports de blé représentant 25 p. 100 de la matière sèche totale, le rapport $\mathrm{C} 2 / \mathrm{C} 3$ dans le rumen est de 3,42 pour le blé entier et 2,63 pour le blé broyé, le $\mathrm{pH}$ enregistrant une baisse de 0,6 point (Coulon et al., 1985).

WaLdo (1973) montre que pour une proportion donnée de céréales dans la ration, la part du C3 augmente lorsque le niveau alimentaire s'accroît. Il en est de même si la proportion de céréales apportée augmente mais les différentes céréales n'ont pas le même effet : le mélange des A.G.V. du rumen varie peu tant que le maïs grain ne dépasse pas 60 p. 100 de la ration alors qu'à 45 p. 100 l'orge favorise la production de C3 (Thivend \& Journet, 1970). Dans les essais in vitro de Varner \& Woods (1975) la substitution du maïs par du blé accroît la production d'acide lactique qui est multiplié par 7 , soit $88 \mu \mathrm{g} / \mathrm{ml}$ au cours de la première heure après le repas mais revient à des valeurs de 10 à $15 \mu \mathrm{g}$ dès la deuxième heure. Parallèlement, le $\mathrm{pH}$ perd 0,3 point.

Les teneurs en A.G.V., lactate compris, les valeurs du pH ruminal ne sont pas différentes dans les essais de comparaison maïs/blé conduits par Fulton et al. (1979) sur des génisses mais l'ingestion est plus faible chez les animaux consommant le blé. Pour ces auteurs, la production de lactate est temporaire après le repas et tend à disparaître même avec des temps d'adaptation courts.

Odle \& Schaeffer (1987) montrent sur des génisses Holstein de $610 \mathrm{~kg}$ alimentées toutes les heures que la teneur en NH3 nécessaire pour optimiser la dégradation de 
l'orge $(125 \mathrm{mg} / \mathrm{l})$ est largement supérieure à celle enregistrée pour le maïs $(61 \mathrm{mg} / \mathrm{l})$, ce qui conduit ces auteurs à émettre l'hypothèse que la dégradabilité des grains de céréales dans le rumen serait ainsi liée au niveau d'ammoniac. Cette observation n'est pas spécifique au cas des céréales et ERDMAN et al. (1986) ont également mis en évidence, sur des vaches fistulées, que la teneur en ammoniac nécessaire à l'optimisation de la digestion est une fonction de la fermentescibilité de la ration. Cette observation est également à la base du système P.D.I.

Lors de l'introduction de maïs, de blé et même d'avoine dans le régime, les 24 premières heures sont caractérisées par un développement important mais temporaire des bactéries lactiques, des coliformes et de Clostridia perfringens dans le rumen (Allison et al., 1975).

Les résultats des 2 essais rapportés par LEBzIEN et al. (1983) illustrent l'effet d'une substitution isoazotée maïs/blé chez la vache laitière recevant deux niveaux de concentrés $(50$ p. 100 foin +50 p. 100 maïs ou blé et 67 p. 100 foin +33 p. 100 maïs ou blé) Il n'y a pas de différence dans l'efficacité de la synthèse microbienne mesurée au ${ }^{15} \mathrm{~N}$ (30,5 g/kg M.O.F.) car si l'azote du blé est plus dégradé que l'azote du maïs dans le rumen ( 91 et 75 p. 100 respectivement) la digestibilité de la matière organique y est également plus forte pour le blé. Les flux azotés au niveau du duodénum ne sont pas significativement différents mais les contenus de duodénum avec les régimes maïs sont plus riches en acides aminés totaux (71,5 p. 100) qu'avec les régimes blé (63,8 p. 100).

\section{Au niveau de l'intestin}

La synthèse bibliographique de Owens et al. (1986) met en évidence une corrélation inverse entre digestibilité de l'amidon dans le rumen et digestibilité intestinale $(\mathrm{r}=-0,75)$. Pour le maïs 53 p. 100 de la qualité d'amidon arrivant dans l'intestin grêle y disparaît mais la disparition pour l'ensemble de l'intestin atteint 72 p. 100 ce qui montre une activité fermentaire non négligeable au niveau du gros intestin. Ces observations sont également applicables au sorgho mais les valeurs de digestibilité sont très inférieures à celles enregistrées pour le maïs. Les travaux de MACALLAN \& LEwIS (1985) montrent que lorsque des jeunes génisses ingìrent du maïs ou du blé broyé l'amidon de blé est mieux digéré (90 p. 100) dans l'intestin grêle que l'amidon de maïs (75 p. 100).

Une limite à la capacité enzymatique de dégradation a été montrée dans le cas d'infusion directe dans l'intestin (OwENs et al., 1986) mais à des doses très supérieures à celles obtenues dans les rations courantes. Sur génisses, l'arrivée au niveau intestinal de $1,287 \mathrm{~kg}$ d'amidon ne permet pas d'observer cette limite (l'amidon de maïs est digéré à 54,6 p. 100) (revue de OWENs et al., 1986).

L'activité optimale de l'amylase pancréatique se situe aux environs d'un $\mathrm{pH}$ de 6,8 et une perte d'activité de l'enzyme de 20 p. 100 est enregistrée pour une baisse de 0,5 point du $\mathrm{pH}$. (OWens et al., 1986). Il semble qu'avec des $\mathrm{pH}$ duodénaux bas (inférieur à 2,5 ) l'intestin soit à même de faire jouer un fort pouvoir tampon par saturation en bicarbonates (Owens et al., 1986). Naturellement, pour des $\mathrm{pH}$ au niveau du duodénum proximal proches de 2,5 à 3 , le flux a un $\mathrm{pH}$ voisin de 6,9 après le canal pancréatique et le pH est basique à la fin de l'intestin grêle. Il semble donc que chez les bovins, moins sensibles aux baisses du pH ruminal que les ovins (Orskov, 1986), le rôle des tampons ne soit pas mis en évidence de façon claire. L'activité de l'amylase 
peut être \pm bloquée par les tannins et certains dérivés de la lignine (MaHMouzadeH et al., 1985), ce qui expliquerait les valeurs inférieures obtenues pour la digestibilité du sorgho.

D'autres paramètres tels qu'une limite dans le rythme d'absorption du glucose au niveau intestinal (mais cette hypothèse est peu vraisemblable car le niveau d'absorption est encore de 100 p. 100 pour des apports de glucose de $1,6 \mathrm{~kg}$ de glucose par jour chez la vache laitière qui peut absorber au niveau intestinal jusqu'à $3 \mathrm{~kg}$ de glucose) (MacAllan \& LewIS, 1985), un transit accéléré, une structure de grain trop peu altérée peuvent diminuer la digestibilité intestinale. L'accroissement du niveau protéique et de la teneur en calcium entraînent souvent une amélioration de la digestibilité de l'amidon de maïs (OWENs et al., 1986).

Pour les protéines, et par rapport au rumen, l'écart entre céréales est moins important quand la digestibilité au niveau du tractus digestif entier est prise en compte (respectivement 61-66-68 p. 100 pour le sorgho, le maïs et l'orge). L'intestin aurait donc pour effet de gommer partiellement les différences mises en évidence au niveau du rumen.

En définitive, à partir du parallélisme observé entre digestion de l'amidon et digestion des protéines dans les essais in vivo de SPICER et al. (1986), il est possible que les différences de digestibilité enregistrées entre céréales soient plus liées à la nature de leurs protéines mais surtout aux interactions protéines-amidon qu'à des caractéristiques physico-chimiques spécifiques de la fraction amylacée (SPICER et al., 1986).

\section{Incidences métaboliques}

Par ce jeu partiel de compensation entre digestion dans le rumen et digestion dans l'intestin, la digestibilité de l'amidon sur l'ensemble du tractus digestif est relativement peu différente, quelle que soit la céréale utilisée, sa nature et sa forme, sauf peut-être avec les grains insuffisamment broyés (THIVEND, 1981).

Malgré tout, le facteur discriminant le mieux l'utilisation de l'amidon entre céréales est sans doute le pourcentage d'amidon échappant à la dégradation dans le rumen (THeurer, 1986) : si davantage d'amidon échappe à la digestion dans le rumen, la dégradabilité globale peut diminuer mais l'utilisation énergétique peut être supérieure. En effet, la digestion de l'amidon dans l'intestin permet un apport énergétique supérieur de 42 p. 100 à l'apport énergétique généré par la digestion ruminale de la même quantité d'amidon (OWENS et al., 1986). La digestion de l'amidon au niveau intestinal productrice de glucose serait donc plus favorable qu'une fermentation microbienne productrice d'acides gras volatils.

C'est ce que traduit l'équation :

efficacité $=0,159$ digestion amidon dans le rumen $+0,227$ digestion amidon dans l'intestin, proposée par Owens et al. (1986) qui relie l'efficacité alimentaire à la digestion dans le rumen et dans l'intestin. Cette remarque permet également d'expliquer la supériorité du roulage des céréales sur le broyage (THEURER, 1986) et étaye les propositions d'utilisation de céréales en mouture grossière (de $250 \mathrm{um}$ à $1 \mathrm{~mm}$ ) qui, optimale au plan énergétique, permet d'éviter le développement de l'acidose et des effets dépressifs sur l'appétit (KIM \& OWENs, 1985). L'influence des interactions fourrages-concentrés qui sont nombreuses a récemment fait l'objet d'une revue bibliographique très complète (Doreau, 1988). 


\section{Utilisation du blé dans la ration des ruminants laitiers}

\section{Comparaison blé - autres céréales en interaction avec la nature des fourrages}

Les résultats des essais de comparaison sont répertoriés dans le tableau 1.

\section{TABleaU 1}

Comparaison blé-céréales dans la ration des vaches laitières.

Comparison of wheat and other cereals for dairy cows.

\begin{tabular}{|c|c|c|c|c|c|c|c|}
\hline \multirow{2}{*}{$\begin{array}{l}\text { Auteurs } \\
\text { Authors }\end{array}$} & \multirow{2}{*}{$\begin{array}{c}\text { Ration de base } \\
\text { Roughage }\end{array}$} & \multirow{2}{*}{$\begin{array}{l}\text { Céréales } \\
\text { Cereals }\end{array}$} & \multicolumn{2}{|c|}{ Quantités intégrées / Intake } & \multirow{2}{*}{$\begin{array}{l}\text { Q. Lait }(\mathrm{kg}) \\
\text { Milk }(\mathrm{kg})\end{array}$} & \multirow{2}{*}{$\begin{array}{l}\text { TB } \% \text { } \\
\text { Fat }\end{array}$} & \multirow{2}{*}{$\begin{array}{l}\text { TP } \% \\
\text { Protein }\end{array}$} \\
\hline & & & $\begin{array}{l}\text { Total }(\mathrm{kg}) \\
\text { Total }(\mathrm{kg})\end{array}$ & $\begin{array}{l}\text { Concentré } \% \\
\text { Concentrate } \%\end{array}$ & & & \\
\hline $\begin{array}{c}\text { HoDEN et al. } \\
1974\end{array}$ & $\begin{array}{l}\text { Foin / Hay } \\
\text { Pulpes betterave } \\
\text { Beet pulps } \\
\text { Ensilage maïs } \\
\text { Maize silage }\end{array}$ & $\begin{array}{l}\text { Maïs / Maize } \\
\text { Blé / Wheat } \\
\text { Orge / Barley }\end{array}$ & $\begin{array}{l}14,5 \\
14,6 \\
14,6\end{array}$ & $\begin{array}{l}26 \\
28 \\
27\end{array}$ & $\begin{array}{l}23,4 \\
23,5 \\
22,9\end{array}$ & $\begin{array}{l}37,9 \\
38,7 \\
34,9\end{array}$ & $\begin{array}{l}33,4 \\
34,9 \\
33,0\end{array}$ \\
\hline $\begin{array}{c}\text { FALDET } \text { et } a l . \\
1986 a\end{array}$ & $\begin{array}{l}\text { Foin luzerne } \\
\text { Lucerne hay }\end{array}$ & $\begin{array}{l}\text { Blé / Wheat } \\
\text { Maïs / Maize } \\
\text { Blé } 60 \text { / Maïs } 40\end{array}$ & $\begin{array}{l}17,8 \\
20,1 \\
18,1\end{array}$ & $\begin{array}{l}55 \\
55 \\
55\end{array}$ & $\begin{array}{l}29,1 \\
30,5 \\
29,7\end{array}$ & $\begin{array}{l}25,6 \\
25,3 \\
28,2\end{array}$ & $\begin{array}{l}28,3 \\
28,2 \\
27,4\end{array}$ \\
\hline $\begin{array}{c}\text { FALDET et al. . } \\
1986 \mathrm{~b}\end{array}$ & $\begin{array}{l}\text { Ensilage Sorgho } \\
\text { + céréales : } \\
\text { Sorghum silage } \\
\text { + cereals } \\
\text { Ration complète } \\
\text { Complete diet }\end{array}$ & $\begin{array}{l}\text { Blé / Wheat } \\
\text { Maïs / Maize }\end{array}$ & 24,6 & 55 & 27,2 & 38,1 & $\begin{array}{l}31,5 \\
31,5\end{array}$ \\
\hline${ }_{1986}^{\text {MoRAN . . . . }}$ & $\begin{array}{l}\text { Foin luzerne } \\
\text { Lucerne hay } \\
\text { Ensilage avoine } \\
\text { Oat silage } \\
\text { Ration complète } \\
\text { Complete diet }\end{array}$ & $\begin{array}{l}\text { Blé / Wheat } \\
\text { Avoine / Oat } \\
\text { Orge / Barley }\end{array}$ & $\begin{array}{l}18,1 \\
17,7 \\
16,9\end{array}$ & $\begin{array}{l}60 \\
60 \\
60\end{array}$ & $\begin{array}{l}24 \\
25,1 \\
22,9\end{array}$ & $\begin{array}{l}41,9 \\
47,2 \\
45,4\end{array}$ & $\begin{array}{l}38,4 \\
31,2 \\
35,2\end{array}$ \\
\hline $\begin{array}{c}\text { NALSEN et al. . } \\
1987 \mathrm{a}\end{array}$ & $\begin{array}{l}\text { Foin luzerne } \\
\text { Lucerne hay }\end{array}$ & $\begin{array}{l}\text { Mais / Maize } \\
\text { Blé / Wheat }\end{array}$ & $\begin{array}{l}24,3 \\
23,0\end{array}$ & $\begin{array}{l}50 \\
50\end{array}$ & $\begin{array}{l}34,5 \\
32,9\end{array}$ & $\begin{array}{l}31,8 \\
32,2\end{array}$ & $\begin{array}{l}28,7 \\
28,6\end{array}$ \\
\hline $\begin{array}{c}\text { NALSEN } \text { et al. . . } \\
1987 \mathrm{~b}\end{array}$ & $\begin{array}{l}\text { Ensilage de } \\
\text { Sorgho } \\
\text { + céréales : } \\
\text { Sorghum silage } \\
\text { + cereals } \\
\text { Ration complète } \\
\text { Complete diet }\end{array}$ & $\begin{array}{l}\text { Maï / Maize } \\
\text { Blé + AA } \\
\text { Wheal + AA } \\
\text { Blé + Protéine } \\
\text { Wheat + Protein }\end{array}$ & $\begin{array}{l}26,4 \\
26,7\end{array}$ & $\begin{array}{l}55 \\
55\end{array}$ & $\begin{array}{l}29,0 \\
30,2\end{array}$ & $\begin{array}{l}37,6 \\
37,8\end{array}$ & $\begin{array}{l}33,1 \\
33,5\end{array}$ \\
\hline
\end{tabular}




\section{a) Effet sur l'ingestion}

L'introduction du blé en substitution à de l'orge ou du maïs n'a pas eu d'effet sur l'ingestion dans les essais de Hoden et al. (1974) : les quantités totales ingérées sont faibles $(14,6 \mathrm{~kg} / \mathrm{j} /$ vache $)$ et le concentré ne représente que $27 \mathrm{p} .100 \mathrm{du}$ total ingéré soit $4 \mathrm{~kg}$ de matière sèche en moyenne. Ces conditions expérimentales expliquent sans doute l'absence de variation dans l'ingestion. Pour Moran (1986) l'ingestion volontaire de vaches, recevant à volonté une ration complète constituée de foin de luzerne et d'ensilage d'avoine ( 40 p. 100 de la M.S.) et de 60 p. 100 de blé ou d'avoine ou d'orge, ne subit pas de variation significative même si la tendance est favorable au blé (tabl. 1). Les concentrations en M.O., en amidon et en azote de la ration blé sont plus fortes que pour les rations orge ou avoine ; les teneurs en cellulose brute, N.D.F. et A.D.F. sont plus faibles d'où une digestibilité meilleure et des quantités de M.O.D. ingérées significativement plus fortes. Ces résultats sont toutefois à considérer avec prudence compte tenu de la brièveté de la période expérimentale ( 3 semaines de mesure) et du faible nombre de vaches (6 par régime). Dans les essais de FALDET et al. (1986 a et b) ( 2 fois 12 V.L. avec un schéma expérimental en inversion) 4 concentrés isoénergétiques et isoazotés mais qui diffèrent par les proportions de maïs et de blé sous forme broyée (de 0 à 80 p. 100 de blé) sont comparés. Quand le concentré représente 55 p. 100 de la M.S. totale ingérée tout apport supplémentaire de blé dans le concentré réduit et l'ingestion de foin de luzerne et l'ingestion du concentré. Les écarts sont moins élevés si le concentré est distribué en ration complète avec de l'ensilage de sorgho : l'effet négatif du blé serait alors tamponné grâce à l'allongement de la période d'ingestion. Malgré le niveau élevé de blé distribué (jusqu'à $8 \mathrm{~kg} / \mathrm{j}$ en 2 repas) aucun trouble d'ingestion n'est signalé mais le concentré était enrichi en bicarbonates. Dans des conditions expérimentales proches (foin de luzerne +50 p. 100 de concentré, substitution maïs/blé, 2 repas $/ \mathrm{j}$, même niveau d'ingestion $=24 \mathrm{~kg} \mathrm{M.S.,} \mathrm{bicarbonate} \mathrm{dans} \mathrm{le}$ concentré) NALSEN et al. (1987 a) enregistrent une baisse d'ingestion qui affecte dans les mêmes proportions le concentré et le fourrage. Cette variation d'ingestion ne se répercute pas sur le pH du rumen. Un apport supplémentaire d'azote dans le concentré blé (passage de 12,1 p. 100 à 15,1 p. 100 de M.A.T.) ne permet pas de retrouver les niveaux d'ingestion obtenus avec le maïs. Dans un second essai NALSEN et al. (1987 b) montrent que le passage à la ration complète avec de l'ensilage de sorgho annule les écarts entre maïs et blé confortant ainsi les observations faites par FALDET et al. (1986 b). Criberro et al. (1979), dans un essai avec des animaux à faible niveau de production $(12,5 \mathrm{~kg})$ mais avec des rations à 70 p. 100 de concentrés $(57$ à 77 p. 100 de blé dans le concentré), notent également une diminution de l'ingestion lorsque la proportion de blé dans le concentré augmente.

En définitive, les effets négatifs sur l'ingestion enregistrés lors de la substitution de blé au maïs concernent des rations à base de foin de luzerne, avec des pourcentages de concentrés supérieurs à $50 \mathrm{p}$. 100 et une distribution du concentré en 2 repas $/ \mathrm{j}$. Les essais répertoriés sont toutefois trop peu nombreux pour conclure objectivement dans d'autres conditions expérimentales.

\section{b) Effet sur la production de lait}

L'absence d'essais concernant la comparaison blé - autres céréales avec des rations à base d'ensilage de maïs ou au pâturage est à souligner. KAISER et al. (1982) montrent que pour 50 vaches au pâturage la réponse marginale est supérieure pour l'apport d'un concentré blé + soja $(80 / 20)$ comparé à un apport de blé seul $(1,15 \mathrm{~kg}$ de lait/kg de 
concentré contre $0,9 \mathrm{~kg} / \mathrm{kg}$ ). L'apport supplémentaire de $3 \mathrm{~kg}$ de blé à des vaches au pâturage (mélange R.G.I. + trèfle blanc) n'a aucun effet bénéfique sur la production dans l'essai de Doвos et al. (1987). Compte tenu du niveau de production (21 kg) des animaux et du faible apport de concentré, ce résultat paraît normal.

Tommervik \& WALDERN (1969) avaient comparé des rations contenant $470 \mathrm{~g} / \mathrm{kg}$ de M.S. de blé, d'orge, d'avoine, de maïs ou de sorgho distribués sous forme granulée. Ces conditions expérimentales expliquent en partie que les écarts entre régimes (vitesse de dégradation dans le rumen, digestibilité intestinale) ne se sont pas exprimés et que la production a été identique. Pour Jeffery et al. (1976) il n'y a pas d'écart entre ces 5 céréales mais leur essai est réalisé avec des vaches au pâturage et $3 \mathrm{~kg}$ seulement de concentré : l'absence de réponse est donc normale.

La comparaison maïs/blé/orge n'a pas montré de différence sur la production et la composition du lait dans les essais de Hoden et al. (1974). Là aussi, les conditions expérimentales (moins de 30 p. 100 de céréales dans la ration diversifiée, pas de variation de l'ingestion) permettent d'expliquer l'égalité entre les régimes. MoRAN (1986) pour des apports bien supérieurs (60 p. 100 de céréales) met en évidence une tendance non significative à la supériorité du blé comparé à l'orge $(+1,1 \mathrm{~kg}$ de lait, +3 points de taux azoté, $-3,5$ points de taux butyreux). Mais c'est l'avoine qui donne la meilleure production. La proportion importante de blé et d'orge utilisée peut avoir limité l'apport de fibres (293 et $255 \mathrm{~g} / \mathrm{kg}$ de N.D.F.) dans le rumen et donc les fermentations. L'avoine, plus riche en cellulose brute et en lipides, a permis de produire plus de lait mais sa teneur en protéines est particulièrement faible (tabl. 1). La richesse relative du lait produit par les vaches recevant le blé est à mettre en relation avec le niveau de M.O.D. ingéré. Pour FALDET et al. (1986 a) la production de lait diminue régulièrement lorsque la teneur en blé augmente dans le concentré reproduisant ainsi les variations de l'ingestion. Le taux butyreux est toujours faible (tabl. 1) mais ceci peut s'expliquer par les faibles apports de cellulose brute dans la ration (< à 17 p. 100) et il a tendance à diminuer lorsque l'apport de blé augmente. Avec la ration complète ensilage de sorgho + blé ou maïs l'étalement de l'ingestion minore les écarts enregistrés (FALDET et al., $1986 \mathrm{~b}$ ). Seule la production de lait diminue lorsque le blé remplace le maïs dans le concentré distribué dans les essais de Nalsen et al. (1987 a). Dans un second essai, avec une ration complète ensilage de sorgho/concentré (45/55), NALSEN et al. (1987 b) montrent que pour obtenir des performances similaires à celles enregistrées avec un concentré maïs, le concentré à base de blé doit comporter des sources azotées peu fermentescibles car l'adjonction d'acides aminés en complément au concentré blé ne permet pas d'obtenir les mêmes performances.

L'ensemble de ces résultats ayant été obtenus au cours de comparaisons conduites sur des périodes relativement courtes (entre 6 et 12 semaines) seules des tendances pourront être dégagées. Il semble donc que la nature de la céréale n'intervienne sur les performances des vaches laitières que dans la mesure où leur apport représente au moins 50 p. 100 de la matière sèche totale et que cet apport est réalisé en 2 repas $/ \mathrm{j}$. Le passage à la ration complète, le passage sous le seuil des $50 \mathrm{p} .100$ de concentrés annulent la majeure partie des écarts enregistrés.

\section{c) Influence de la forme de présentation}

L'essai de Coulon et al. (1985) montre clairement que les vaches laitières ne sont pas capables de valoriser efficacement du blé présenté en grains entiers. Par contre, 
l'utilisation sous forme broyée, jusqu'à 25 p. 100 du total de la ration, permet l'obtention de résultats satisfaisants (tabl. 2). L'effet positif du broyage sur la production laitière est à mettre en relation avec l'amélioration de la digestibilité de la matière sèche et de la matière organique lorsque l'on passe de la forme entière (respectivement $61,9$ et 63,6 p. 100$)$ à la forme broyée $(72,6$ p. 100 et 74,9 p. 100$)$. Fulkerson \& Michell (1985) notent également la supériorité du blé aplati par rapport au blé entier chez des vaches recevant un régime mixte pâturage et ensilage d'herbe.

\section{Tableau 2}

Influence de la forme d'apport du blé sur les performances des vaches laitières.

Effect of wheat processing on dairy cows performance.

\begin{tabular}{|c|c|c|c|}
\hline & \multicolumn{2}{|c|}{$\begin{array}{l}\text { Forme d'apport } \\
\text { Wheat processing }\end{array}$} & Conditions de l'essai / Trial conditions \\
\hline $\begin{array}{l}\text { MS ing. / Intake } \\
\text { Emaïs / maize sil . . . . . } \\
\text { blé / wheat . . . . . } \\
\text { lait / milk }(\mathrm{kg}) \ldots \ldots \\
\text { TB } \% \text {. fat } \ldots \ldots \\
\text { TP } \% \text { / protein . . . . } \\
\text { Perte PV / Weight loss. . }\end{array}$ & $\begin{array}{r}9,8 \\
3,5 \\
18,3 \\
41,1 \\
29,8 \\
-\quad 31\end{array}$ & $\begin{array}{c}\begin{array}{c}\text { broyé } \\
\text { ground }\end{array} \\
\\
8,8 \\
3,9 \\
23,0 \\
38,9 \\
30,6 \\
-\quad 14\end{array}$ & $\begin{array}{l}\text { Coulon et al. } 1985 \\
\text { Ensilage maïs à volonté }+ \text { urée }+0,5 \mathrm{~kg} \text { tourteau } \\
\text { tanné / Maize silage }+ \text { urea }+0,5 \mathrm{~kg} \text { soya } \\
\text { Vaches en fin de gestation et en début de lacta- } \\
\text { tion / Pregnant cows and cows in early lactation }\end{array}$ \\
\hline $\begin{array}{l}\text { MS ing. / Intake } \\
\text { Emaïs / maize sil . . . . . } \\
\text { blé / wheat } \ldots \ldots \\
\text { lait / milk }(\mathrm{kg}) \ldots \ldots \\
\text { TB } \% \text {. . fat . . . . . } \\
\text { TP \% / protein . . . } \\
\text { GMQ / Daily weight gain }\end{array}$ & $\begin{array}{l}13,8 \\
4,0 \\
25,8 \\
36,5 \\
29,6 \\
58 \mathrm{~g} / \mathrm{j}\end{array}$ & $\begin{array}{c}\text { broyé } \\
\text { ground } \\
\\
13,9 \\
4,1 \\
25,9 \\
36,3 \\
29,8 \\
299\end{array}$ & $\begin{array}{l}\text { LAURENT } \\
\text { Ensilage de maïs ad lib }+ \text { urée }+0,5 \mathrm{~kg} \text { tourteau } / \\
\text { Maize sil. }+ \text { urea }+0,5 \mathrm{~kg} \text { soya bean meal } \\
\text { Carré latin } 4 \times 5 \text { après le pic } / \text { Latin square. Cows } \\
\text { after the peak of lactation }\end{array}$ \\
\hline $\begin{array}{l}\text { Blé / wheat intake } \ldots \ldots \\
\text { lait / milk }(\mathrm{kg}) \ldots \ldots \\
\text { TB } \% \text { / fat . . . . . . . } \\
\text { TP \% / protein . . . } \\
\text { GMQ / Daily weight gain }\end{array}$ & $\begin{array}{l}\text { aplati } \\
\text { rolled } \\
6,8 \\
31,2 \\
43,2 \\
29,1 \\
-308\end{array}$ & $\begin{array}{c}\text { broyé } \\
\text { ground } \\
6,9 \\
29,5 \\
40,1 \\
28,3 \\
-325\end{array}$ & $\begin{array}{l}\text { ChENaIs (ITEB) } \\
\text { Vaches en début de lactation / Cows at early } \\
\text { lactation } \\
\text { Ensilage de maìs + urée } 10 \text { semaines / Maize si- } \\
\text { lage + urea } 10 \text { weeks }\end{array}$ \\
\hline
\end{tabular}

Dans des essais de courte durée (période de 3 semaines, carré latin) les différences de granulométrie entre un blé broyé $(1,9$ p. 100 des particules $>$ à $2,5 \mathrm{~mm}, 59,3$ p. 100 entre 1 et $2,5 \mathrm{~mm}, 39,7$ p. $100<$ à $1 \mathrm{~mm})$ et un blé aplati $(63,1$ p. 100 et 32 p. 100 et 4,9 p. 100) n'ont pas fait varier significativement la production et la composition du lait (LAURENT, non publié). Mais le concentré associé à l'ensilage de maïs ne représentait que 30 p. 100 de l'apport total de M.S. Des résultats d'expériences menées par 
l'I.T.E.B. (ChEnAIs, non publié) montrent que sous forme broyée $4,5 \mathrm{~kg}$ de blé (soit $6,5 \mathrm{~kg}$ de concentrés) pénalise la production comparativement à un apport de blé aplati en début de lactation (tabl. 2). Avec des animaux en phase descendante de lactation qui ingèrent en moyenne $5,2 \mathrm{~kg}$ de concentré (soit $3,4 \mathrm{~kg}$ de blé) la forme de présentation n'a aucune influence sur les performances. Les essais sur génisses de Bergner \& Weissbach (1983), ceux de Toland (1976) et de Hamilton \& Maden (1980) avaient également montré une digestibilité supérieure (entre 16 et 30 points d'écart pour le C.U.D. de la M.O.) pour du blé présenté sous forme broyée ou aplatie comparée à du blé entier.

Pour MORAN (1986), l'aplatissage de l'avoine n'améliore pas significativement la production laitière, contrairement aux effets marqués enregistrés avec le blé. La vache utilise bien l'avoine entière et dans ces essais, après le roulage, le T.P. est légèrement amélioré alors que le T.B. baisse. Le pH est identique avec les deux formes de présentation mais le rapport $\mathrm{C} 2 / \mathrm{C} 3$ est largement supérieur avec l'avoine entière $(3,84$ contre 3,24) ce qui peut expliquer l'évolution inverse des taux. LaKsesvela (1982) a montré que pour l'orge un broyage fin ( $83 \mathrm{p} .100$ des particules $<$ à $1 \mathrm{~mm})$ assure une production supérieure $(+2,7 \mathrm{~kg}$ de lait $4 \mathrm{p}$. 100) à celle obtenue pour l'orge broyée grossièrement $(55 \mathrm{p} .100$ des particules $<$ à $1 \mathrm{~mm})$. Ces résultats peuvent paraître surprenants mais la composition de la ration de base $(7 \mathrm{~kg}$ de paille traitée à la soude $+5 \mathrm{~kg}$ d'ensilage d'herbe), et le faible niveau d'apport du concentré $(5 \mathrm{~kg})$ peuvent les expliquer.

\section{d) Limites d'utilisation}

Chez la vache laitière, FALDET et al. (1986 a et b) n'ont observé ni refus anormaux, ni acidose clinique même pour un niveau d'ingestion moyen de $8 \mathrm{~kg}$ de blé et malgré une teneur en cellulose brute de la ration inférieure au seuil de 17 p. 100 ; cette teneur a sans doute rejailli sur les taux butyreux qui sont faibles (à peine 26 p. 1000 ). MoRAN (1986) avance les mêmes conclusions pour une consommation de $10 \mathrm{~kg}$ de blé mais distribué en ration complète. Coulon et al. (1985) ont pour leur part enregistré des chutes d'ingestion fréquentes chez des vaches consommant $5 \mathrm{~kg}$ de blé en complément à de l'ensilage de maïs mais ces problèmes n'ont été accompagnés d'aucun trouble sanitaire visible. ODDY (1978) fait état d'acidose systématique chez la brebis lorsque le blé représente 85 p. 100 de la ration mais ces conditions d'expérimentation paraissent plus que limites.

En fait, les chutes d'ingestion observées sont souvent simultanées avec des baisses $\mathrm{du} \mathrm{pH}$ ruminal. L'infusion d'acide lactique dans le rumen réduit l'ingestion (Fulton et al., 1979) : plus une ration est favorable à la production d'acide lactique, plus la baisse de $\mathrm{pH}$ est potentiellement importante, plus la réduction de l'ingestion peut être forte. Le blé étant plus «acidosique » que le maïs, sa substitution au maïs peut donc conduire à des baisses brutales de l'ingestion. Une substitution blé/sorgho a les mêmes effets (AXE, 1986 ; AxE et al., 1987). Néanmoins, pour des teneurs en blé dans la ration ne dépassant pas $60 \mathrm{p}$. 100, et à condition de ménager une période de transition, les risques d'acidose paraissent peu importants. La sensibilité à ce type d'accident paraît accrue dans le cas où le maïs constitue la majeure partie de la ration de base mais cette observation de Coulon et al. (1985) est à confirmer. 


\section{Conclusions}

En définitive, il ressort de cette synthèse bibliographique que :

* dans le grain, les amidons du blé, de l'orge et de l'avoine sont plus dégradés que ceux du maïs, du sorgho dans le rumen; ces différences de digestibilité enregistrées entre les céréales au niveau du rumen pourraient être plus liées à la nature des protéines du grain et aux interactions protéines*amidon qu'aux caractéristiques intrinsèques de l'amidon;

* la dégradation de l'amidon dans le rumen a tendance à diminuer avec le niveau de fibres dans la ration. Mais la digestibilité dans le rumen des fourrages n'est pratiquement pas perturbée tant que les céréales ne dépassent pas $30 \mathrm{p} .100 \mathrm{de}$ la ration ;

* les différentes céréales n'ont pas le même impact sur le mélange d'A.G.V. du rumen. La composition du mélange A.G.V. ne varie pas tant que le maïs ne dépasse pas 60 p. 100 de la ration mais ce seuil est proche de 45 p. 100 pour l'orge. Par ailleurs, l'utilisation de blé et même d'orge en substitution au maïs ou au sorgho augmente les risques potentiels d'acidose lactique mais ces risques ne sont à envisager que pour des apports élevés de concentrés (au moins 50 p. 100 de la M.S. de la ration).

L'apport énergétique par digestion de l'amidon dans l'intestin étant de 40 p. 100 supérieur à celui obtenu par sa digestion dans le rumen une moindre dégradabilité de l'amidon dans le rumen peut donc favoriser un accroissement de l'apport énergétique et ce d'autant plus que la dégradabilité du fourrage associé risque d'être moins diminuée ;

* en pratique, l'utilisation du blé comme concentré chez la vache laitière ne présente pas d'inconvénient majeur tant que la proportion de blé dans la ration ne dépasse pas 25 à 30 p. 100 de la M.S. (soit environ 4 à $5 \mathrm{~kg}$ ) et à condition de l'utiliser sous forme broyée ou aplatie et de ménager des périodes de transition suffisante. Cette remarque générale peut être modulée en fonction :

— de la nature de la ration de base : si des consommations de 8 à $10 \mathrm{~kg}$ de blé sont possibles sans trouble sanitaire apparent avec des rations à base de foin il semble qu'avec l'ensilage de maïs la fréquence des chutes d'ingestion augmente rapidement audelà de $5 \mathrm{~kg}$,

- des modalités de distribution : le blé entier est toujours mal utilisé. Pour des apports de 8 à $10 \mathrm{~kg}$ la distribution de blé en 2 repas par jour ou en ration complète entraîne un niveau d'ingestion et une production laitière inférieurs à ceux obtenus avec du maïs grain. Il convient de rester prudent quant à ces conclusions dans la mesure où la majorité des résultats découlent d'essais relativement courts.

Reçu en février 1988. 


\section{Summary}

\section{Review : Utilization of wheat grain and other cereals in diets for dairy cows}

According to its digestible and metabolizable energy values wheat ranks second after maize among cereals. Despite in many experiments it has been shown that it is possible to use wheat as the only grain for beef cattle or ewes, wheat was rarely used alone for dairy cows.

The review reported below compares responses of dairy cows to diets containing different types of cereal grain and particularly wheat. Wheat contains only 2 p. 100 fibre, is as digestible as maize but surpasses all cereals in its protein content. Like other cereals, the protein in wheat grain is of relatively poor quality.

Wheat showed a greater degradability or fermentation rate in the rumen than maize : differences were presumed to be related with the characteristics of the grain proteins. The $\mathrm{C} 2 / \mathrm{C} 3$ ratio was generally wider with wheat than with maize.

Intake of both concentrate and lucerne hay was affected by the substitution of wheat to maize when the proportion of concentrate is above 50 p. 100 in the diet (table 1). Similar yields of F.C.M. have been reported earlier in cows given wheat, barley, oat, sorghum or maize. When compared with maize, milk production response and milk composition did not decline with wheat so much that the amount of concentrate dry matter remained under 50 p. 100 of total D.M. intake (table 1).

Physical processing of cereal grains is often associated with improvements in efficiency of utilization. Rolling or grinding the wheat always improved milk production as compared with whole wheat (table 2) but we have no answer to the question: which medium degree of grinding grain is best for dairy cattle ? Finally, wheat can be incorporated in a nutrition scheme for dairy cows when some management factors are used.

Key words : Cereals, wheat, dairy cow, milk.

\section{Références bibliographiques}

Allison M.J., Robinson J.M., Dougherty R.W., Bucklin J.A., 1975. Grain overload in cattle and sheep. Changes in microbial populations in the caecum and rumen. Amer J.Veter. Res., 36, 181-185.

AXE D.E., 1986. Effect of wheat and high moisture sorghum grain fed singly and in combination in rumen fermentation, solid and liquid flow, site and extent of digestion and feedlot performance of cattle. Diss. Abstr. Int., 47, 1804 B.

Axe D.E., Bolsen K.K., Harmon D.L., Lee R.W., Milliken G.A., Avery T.B., 1987. Effect of wheat and high moisture sorghum grain fed singly and in combination on ruminal fermentation, solid and liquid flow, site and extent of digestion and feeding performance of cattle. $J$. Anim. Sci., 64, 897-906.

Bergner E., Weissbach F., 1983. Zum Einfluss der Zerkleinerungsgrades auf die Verdaulichkeit von Getreidekörnern. 1. Untersuchungen mit Weizen und Gerste an Rindern. Archiv. Tierernäh., 33, 241-250.

Cheftel J.C., Cuq J.L., Lorient D., 1985. Les Protéines de blé in « Protéines alimentaires », Tec. Doc. Lavoisier éditeur, pp. 204-222.

Coulon J.B., Didi M., Remond B., 1985. Utilisation du blé par les vaches laitières : influence de la forme de présentation. Bull. Tech. C.R.Z.V. Theix, INRA, 62, 27-33.

Cribeiro T.C., Elias A., Denis I., 1979. Substitution levels of maize by wheat in concentrates and their effects on milk production and composition. Cuban J. Agric. Sci., 13, 19-24. 
Dobos R.C., Fulkerson W.J., Michel P.J., 1987. Effect on milk production of feeding wheat supplements to grazing dairy cows in early lactation. Aust. J. Exp. Agric., 27, 41-44.

DOREAu B., 1988. Influence de la nature du concentré, amidon ou paroi, sur la digestion. In L'utilisation des céréales par les vaches laitières, 24 p., I.T.E.B. Paris.

Erdman R.A., Proctor G.H., Vandersall J.H., 1986. Effect of rumen ammonia concentration on in situ rate and extent of digestion of feedstuffs. J. Dairy Sci., 69, 2312-2318.

Faldet M.A., Bush L.J., Adams G.D., 1986 a. Effect of different levels of wheat in concentrate mixture on production responses of lactating dairy cows fed sorghum silage as the only forage. Oklahoma Anim. Sci. Res. Rep., 66-70.

FALDET M.A., BuSh L.J., AdAMS G.D., 1986 b. Effect of different levels of wheat in concentrate mixture on production responses of lactating dairy cows fed alfafa hay as the only forage. Oklahoma Anim. Sci. Res. Rep., 71-76.

Fulkerson W.J., Michell P.J., 1985. Production response to feeding wheat grain to milking cows. Austr. J. Exp. Agric. Anim. Husbandry, 25, 253-256.

Fulton W.R., Klopfenstein J.J., Britton R.A., 1979. Adaptation to high concentrate diets by beef cattle. Adaptation to corn and wheat diets. J. Anim. Sci., 49, 775-784.

Gallant D.J., Bouchet B., 1986. Ultrastructure of maize starch granule, a review. Food Microstruct., 5, 141-155.

Hamilton D., Maden J.J.L., 1980. Liveweights and ratio digestibilities for steers fed oats or wheat rations at near maintenance level. Austr. J. Exper. Agric. Anim. Husb., 20, 674-677.

HENRY R.J., 1985. A comparison of the non starch carbohydrates in cereals grains. J. Sci. Food Agric., 36, 1243-1253.

Hoden A., Remond B., Journet M., 1974. Utilisation comparée de 3 céréales grain (maïs, blé, orge) par les vaches laitières en début de la lactation. Bull. Tech. C.R.Z.V. Theix, INRA, 16, $17-20$.

JefFery H., Buesnel R.J., O'Neill G.H., 1976. Short-term studies on the response of grazing dairy cows to dietary supplementation. Austral. J. Exper. Agric. Anim. Husb., 16, 445-451.

Kaiser A.G., Ashwood A.M., Detrmann E.B., 1982. Influence of wheat, soya bean meal and sodium bicarbonate supplements on the yield and composition of milk from grazing dairy cows. Proc. Austr. Soc. Anim. Prod., 14, 622 (Abstr.).

KiM Y.K., OWENS F.N., 1985. Starch digestion by feedlot cattle : Influence of roughage and intake level and particle size. Oklahoma Agr. Exp. Sta. MP. 117, p. 298.

Lebzien P., Rohr K., SchaffT H., 1983. Vergleichende Untersuchungen über die N Umsetzungen im Verdauungstrakt bei Milchkühen von Weizen bzw. Körnermaïs. Landbauforschung Volkenrode, $33,57-64$.

LAKSESvela B., 1982. Intensity of grinding on the value of grain for dairy cows. Z. Tierphysiol., Tierernähr. Futtermittelkde., 48, 9-15.

MacAllan A.B., Lewis P.E., 1985. The removal of glucose, lactose, and different starches from the small intestine of steers. Archiv. Tierernähr., 35, 495-505.

Mahmouzadeh H., Muntifering R.B., Mitchell G.E., Tucker R.E., 1985. Starch digestion in the presence of lignin monomers. J. Anim. Sci., 61 (suppl. 1), 483 (Abstr.).

Moran J.B., 1986. Cereal grains in complete diets for dairy cows : a comparison of rolled barley, wheat and oats and of three methods of processing oat. Anim. Prod., 43, 27-36.

Nalsen T., Bush L.J., Adams G.D., 1987 a. Substituting wheat for corn in a concentrate mixture for dairy cows on a weight basis. Oklahoma Exp. Stat., MP. 119, 149-152.

Nalsen T., Bush L.J., Adams G.D., 1987 b. Amino acid supplementation of high wheat concentrate mixture for dairy cows. Oklahoma Exp. Stat., MP. 119, 144-148.

Nalsen T., Bush L.J., Adams G.D., 1987 c. Determination of protein degradation in the rumen and intestines of heifers using a mobile dacron bag technique. Oklahoma Anim. Sci. Res. Rep., 153-156.

Oddy V.H., 1978. Milk production in ewes fed high grain diets. Proc. Austr. Soc. Anim. Prod., 12, 145 .

OdLe J., SchaEfer D.M., 1987. Influence of rumen ammonia concentration on the rumen degradation rate of barley and maize. Br. J. Nutr., 57, 127-138. 
Orskov E.R., 1986. Starch digestion and utilization in ruminants. J. Anim. Sci., 63, 1624-1633.

OWENS F.N., ZINN R.A., KIM Y.K., 1986. Limits to starch digestion in the ruminant small intestine. J. Anim. Sci., 63, 1634-1648.

Popineau V., 1985. Propriétés biochimiques et physicochimiques des protéines de céréales in "Protéines végétales", B. Godon, Tec. Doc. Lavoisier, Paris, pp. 161-210.

RoHr K., 1986. Physiological aspects in the rumen in relation to the level of production in ruminants. Arch. Anim. Nutr., Berlin, 36, 182-192.

Rooney L.W., Pflugfelder R.L., 1986. Factors affecting starch digestibility with special emphasis on sorghum and corn. J. Anim. Sci., 63, 1607-1623.

Sauvant D., Michelet-Doreau B., 1988. Les aliments concentrés. In Alimentation des bovins, ovins, caprins. INRA, Paris, pp. 337-349.

Spicer L.A., Theurer C.B., Young M.C., 1983. Ruminal and postruminal utilization of protein from feed grains by beef steers. J. Anim. Sci., 57 (Suppl. 1), 470 (Abstr.).

Spicer L.A., Theurer C.B., Sowe J., Noon T.H., 1986. Ruminal and postruminal utilization of nitrogen and starch from sorghum grain, corn and barley based diets by beef steers. J. Anim. Sci., 62, 521-530.

Swinkels J.J.M., 1985. Sources of starch, its chemistry and physics. In Starch conversion technology, ed. by G.M.A. Van Beynum, J.A. Roels, Marcel Dekker Inc., New York, pp. 15-46.

Theurer G.B., 1986. Grain processing effects on starch utilization by ruminants. J. Anim. Sci., 63, $1649-1662$.

Thivend P., 1981. Les constituants glucidiques des aliments concentrés et sous-produits. In Prévision de la valeur nutritive des aliments des ruminants. Ed. INRA publications, route de Saint-Cyr, 78000 Versailles, pp. 219-236.

Thivend P., Journet M., 1970. Utilisation digestive de l'amidon du mais chez le ruminant. Ann. Biol. Anim. Bioch. Biophys., 10, 323-326.

Toland P.C., 1976. The digestibility of wheat, barley or oat grain fed either whole or rolled, at restricted levels with hay to steers. Austr. J. Exper. Agric. Anim. Husbandry, 16, 71-75.

Tommervik R.S., Waldern D.E., 1969. Comparative feeding value of wheat, corn, barley, oats, milo and a mixed concentrate ration for lactating cows. J. Dairy Sci., 52, 68-73.

VARNER L.W., WooDS W., 1975. Influence of wheat variety upon in vitro and in vivo lactate levels. J. Anim. Sci., 41, 900-905.

Vérité R., Chapoutot P., Michalet B., Peyraud J.L., Poncet C., 1987. Révision du système des protéines digestibles dans l'intestin (P.D.I.). Bull. Tech. C.R.Z.V. Theix, INRA, 70, 19-34.

Waldo D.R., 1973. Extent and partition of cereal grain starch digestion in ruminants. J. Anim. Sci., 37, 1062-1074.

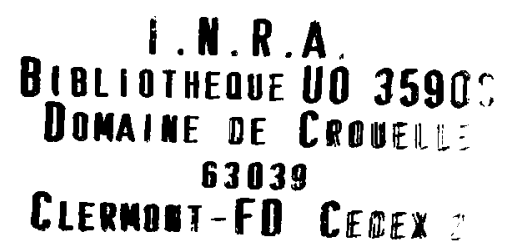

